\title{
Critères d'arrêt de la grossesse en cas de prééclampsie
}

\section{Abstract de la SETHAG, manifestation associée des journées de la Société française de médecine périnatale}

\section{Criteria for stopping a pregnancy in cases of pre-eclampsia}

\section{B. Haddad}

(C) Springer-Verlag France 2011

Reçu le 7 octobre 2011 ; accepté le 13 octobre 2011

Résumé : Les études disponibles pour établir des critères clairs d'arrêt de la grossesse chez une patiente ayant une prééclampsie (PE) sont rares; elles sont dans leur grande majorité soit rétrospectives, soit observationnelles. L'analyse des données de la littérature et les avis d'experts permettent de suggérer des critères d'arrêt de la grossesse, essentiellement dans le cadre de la PE sévère. Certaines des indications de l'arrêt de la grossesse sont maternelles : une hypertension sévère non contrôlée, une éclampsie, un œdème

\section{B. Haddad ( $\square)$}

CHI de Créteil, 40, avenue de Verdun,

F-94010 Créteil cedex, France

e-mail : Bassam.Haddad@chicreteil.fr aigu du poumon, un hématome rétroplacentaire, une oligurie ( $<100 \mathrm{ml} / 4$ heures) persistante malgré un remplissage vasculaire, des signes d'imminence d'une crise d'éclampsie (céphalées ou troubles visuels persistants), une douleur épigastrique persistante, un HELLP syndrome, une insuffisance rénale de novo ou un terme inférieur à 24 semaines d'aménorrhée (SA). D'autres indications sont fœtales : la présence de décélérations prolongées ou variables sévères ou une variabilité à court terme inférieure à $3 \mathrm{~ms}$, un score de Manning inférieur ou égal à 4 à deux reprises, un oligoamnios sévère, une estimation du poids fotal inférieure au cinquième percentile, au-delà de $30 \mathrm{SA}$, une diastole ombilicale artérielle inversée, au-delà de $30 \mathrm{SA}$. En cas de PE peu sévère, l'arrêt de la grossesse doit être envisagé au-delà de 36 SA. 